\title{
Cooperative Differential Space-Time Spreading for the Asynchronous Relay Aided CDMA Uplink Using Interference Rejection Spreading Code
}

\author{
Shinya Sugiura, Member, IEEE, Sheng Chen, Fellow, IEEE, and Lajos Hanzo, Fellow, IEEE
}

\begin{abstract}
This letter proposes a differential Space-Time Coding (STC) scheme designed for asynchronous cooperative networks, where neither channel estimation nor symbol-level synchronization is required at the cooperating nodes. More specifically, our system employs differential encoding during the broadcast phase and a Space-Time Spreading (STS)-based amplify-and-forward scheme during the cooperative phase in conjunction with interference rejection direct sequence spreading codes, namely Loosely Synchronized (LS) codes. Our simulation results demonstrate that the proposed Cooperative Differential STS (CDSTS) scheme is capable of combating the effects of asynchronous uplink transmissions without any channel state information.
\end{abstract}

Index Terms-Asynchronous cooperation, cooperative diversity, differential space-time spreading, loosely synchronized codes.

\section{INTRODUCTION}

C OOPERATIVE Space-Time Coding (STC) schemes [1] were proposed to achieve the best attainable diversity gain of uncorrelated elements, where a collection of single-antenna-aided nodes act as a virtual antenna array, having widely separated distributed antenna elements. On the other hand, attaining a high cooperative space-time diversity gain in a practical relay-aided network imposes further challenges. Firstly, many of the previously-proposed cooperative STC schemes assumed that perfect Channel State Information (CSI) of the Source-Relay (SR) links and/or of the Relay-Destination (RD) links is available at the destination receiver. However, the rapidly changing topology of vehicles travelling at high speeds makes it challenging to acquire accurate CSI, which results in a severe degradation of the achievable performance. By contrast, inspired by the differential STC philosophy developed for collocated MIMO systems [2], a number of Cooperative Differential STCs (CDSTCs) were proposed in [3], [4] in order to achieve reliable symbol detection without any CSI.

Another major challenge is the asynchronous nature of relay nodes in the network. The aforementioned cooperative STCs are all based on the assumption of perfect timing synchronization between the relay nodes, although in practice it is difficult

Manuscript received August 27, 2009; revised October 01, 2009. First published October 13, 2009; current version published November 06, 2009. This work was supported by the EU under the auspices of the Optimix project and of the EPSRC UK. The associated editor coordinating the review of this manuscript and approving it for publication was Dr. Tan F. Wong.

S. Sugiura is with the School of Electronics and Computer Science, University of Southampton, Southampton, SO17 1BJ, U.K., on leave from the Toyota Central Research and Development Laboratories, Inc., Aichi, 480-1192, Japan.

S. Chen and L. Hanzo are with the School of Electronics and Computer Science, University of Southampton, Southampton, SO17 1BJ, U.K. (e-mail: 1h@ecs.soton.ac.uk).

Digital Object Identifier 10.1109/LSP.2009.2034236 to acquire accurate symbol-level time synchronization without imposing a high additional synchronization overhead and implementation complexity. As noted in [5], the resultant time synchronization errors impose a significant performance degradation. To this end, a number of asynchronous cooperative STCs were proposed [6], [7], which invoke space-time equalization or multi-carrier transmission techniques, assuming that the perfect CSI and/or the relative transmission delays of the mobiles are available at the destination.

Against this background, we first propose a practical CDSTC scheme designed for asynchronous relay networks, where neither channel estimation nor symbol-level synchronization is required at any of the nodes. More specifically, our system employs differential encoding during the broadcast phase and an Space-Time Spreading (STS)-based [8] amplify-and-forward scheme during the cooperative phase in conjunction with interference rejection spreading codes, namely Loosely Synchronized (LS) spreading codes [9].

\section{SYSTEM OVERVIEW}

Consider a collection of mobile nodes, each having a single antenna element and communicating with each other or with a specific destination node via frequency-flat Rayleigh fading channels, which corresponds to an uplink scenario. We assume the employment of a Time Division aided CDMA (TD-CDMA) based channel allocation scheme, where $N$ DS-CDMA source nodes are supported in each of the time slots with the aid of $N$ unique, user-specific DS-CDMA spreading codes. More specifically, each transmission of the $N$ source nodes is assisted by the preassigned source-specific $N_{\mathrm{R}}$ relay nodes, and the signal transmission involves two phases, i.e., the broadcast phase and the cooperative phase. Additionally, we introduce node-specific delays caused by their time synchronization errors, where the delays of the $n$th source node and that of the associated $k$ th relay node are represented by $\tau_{n}$ and $\tau_{n k}$, respectively. Furthermore, the Channel Impulse Responses (CIRs) of the SR channels, the Source-Destination (SD) channel and the RD channels are described by $h_{n}^{\mathrm{SR}_{k}} e^{j \theta_{n k}^{\mathrm{SR}}}, h_{n}^{\mathrm{SD}} e^{j \theta_{n}^{\mathrm{SD}}}$ and $h_{n}^{\mathrm{RD}} e^{j \theta_{n k}^{\mathrm{RD}}}$, each modeled as complex Gaussian variables with zero means and variances of $\sigma_{\mathrm{SR}_{k}}^{2}, \sigma_{\mathrm{SD}}^{2}$ and $\sigma_{\mathrm{RD}_{k}}^{2}$, respectively. Here, $h_{n}^{\mathrm{SR}}, h_{n}^{\mathrm{SD}}$ and $h_{n}^{\mathrm{RD}}$ are the corresponding fading envelopes, while $\theta_{n k}^{\mathrm{SR}}, \theta_{n}^{\mathrm{SD}}{ }^{n}$ and $\theta_{n k}^{\mathrm{RD}}$ are the uniformly distributed phase-shift components.

Without loss of generality, we focus our attention on the first time slot, where each of the $N$ sources communicates with a certain destination node with the aid of the $N_{\mathrm{R}}$ source-specific cooperating nodes selected from all the nodes, as illustrated in Fig. 1. To be specific, we consider the case of $N_{\mathrm{R}}=4$ relay 


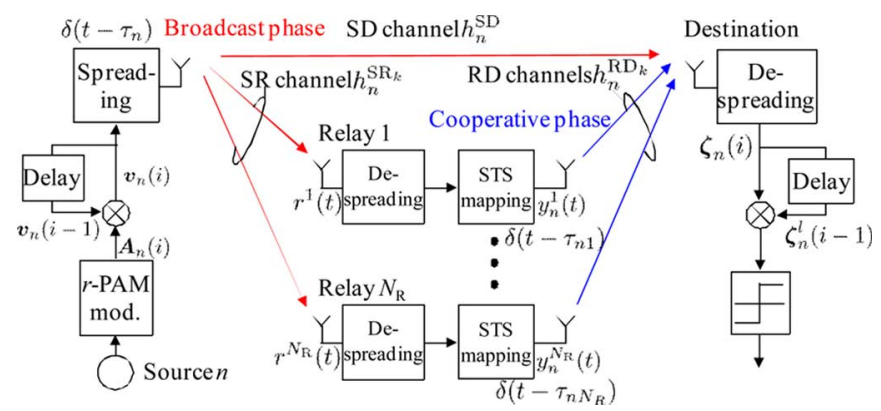

Fig. 1. Dual-phase transmission model of our CDSTS.

nodes, where the resultant maximum space-time diversity order provided by the relay nodes is also four.

\section{A. Transmitted Signal}

During the broadcast phase of Fig. 1, each of the $N$ source nodes transmits the differentially-encoded symbols both to the $N_{\mathrm{R}}=4$ relay nodes and to the destination node. Let the $n$th source node be the source-of-interest in this system model. First, the source bits $b_{n}(q)$ are mapped to $r$-bit PAM-modulated symbols and then the modulated symbols are Serial-to-Parallel $(\mathrm{S} / \mathrm{P})$ converted to the symbol blocks $\boldsymbol{a}_{n}(i)$, each containing four modulated symbols formulated as $\boldsymbol{a}_{n}(i)=\left[a_{n}^{1}(i) a_{n}^{2}(i) a_{n}^{3}(i) a_{n}^{4}(i)\right]^{T}$, where $q$ and $i$ represent the symbol and the block indices, respectively. Then, based on the symbol blocks $\boldsymbol{a}_{n}(i)$, the corresponding differentially-encoded symbol blocks $\boldsymbol{v}_{n}(i)=\left[v_{n}^{1}(i) v_{n}^{2}(i) v_{n}^{3}(i) v_{n}^{4}(i)\right]^{T}$ are generated as follows: [10] $\boldsymbol{v}_{n}(i)=A_{n}(i) \cdot \boldsymbol{v}_{n}(i-1) /\left\|\boldsymbol{v}_{n}(i-1)\right\|$ with

$$
\boldsymbol{A}_{n}(i)=\left[\begin{array}{cccc}
a_{n}^{1}(i) & a_{n}^{2}(i) & a_{n}^{3}(i) & a_{n}^{4}(i) \\
-a_{n}^{2}(i) & a_{n}^{1}(i) & a_{n}^{4}(i) & -a_{n}^{3}(i) \\
-a_{n}^{3}(i) & -a_{n}^{4}(i) & a_{n}^{1}(i) & a_{n}^{2}(i) \\
-a_{n}^{4}(i) & a_{n}^{3}(i) & -a_{n}^{2}(i) & a_{n}^{1}(i)
\end{array}\right]
$$

where the first block $\boldsymbol{v}_{n}(0)=\left[\begin{array}{llll}1 & 1 & 1 & 1\end{array}\right]^{T}$ is transmitted as a reference of the next differentially-encoded block. To relate the block index $i$ to the symbol index $q$, let us define the $q$ th differentially-modulated symbol as $\bar{v}_{n}(q)$, having the relation of $\bar{v}_{n}(4 i+k-1)=v_{n}^{k}(i)$. Furthermore, the time-domain waveform transmitted by the $n$th source can be written as $s_{n}(t)=$ $\sum_{q=-\infty}^{\infty} \bar{v}_{n}(q) \psi_{T_{s}^{\mathrm{S}}}\left(t-q T_{s}^{\mathrm{S}}\right)$, where $\psi_{\alpha}(t)$ is the rectangular waveform, which is defined over the interval $[0, \alpha)$ and $T_{s}^{\mathrm{S}}$ represents the symbol duration of the broadcast phase.

Next, the differentially-modulated symbols $\bar{v}_{n}(t)$ are spread with the aid of the source's node-specific spreading code $c_{n}(t)=\sum_{i=0}^{L_{\mathrm{S}}-1} c_{n i} \psi_{T_{c}}\left(t-i T_{c}\right)$, where $L_{\mathrm{S}}$ is the code length of the spreading code and $T_{c}$ is the chip duration, while $c_{n i}$ represents the $i$ th chip value. Here, we have the relation of $T_{s}^{\mathrm{S}}=L_{\mathrm{S}} T_{c}$. Note that the spreading factor $G_{\mathrm{S}}$ of the spreading code is given by the number of nonzero chips.

Having obtained the transmitted time-domain waveform $y_{n}(t)$ as $y_{n}(t)=\sqrt{P_{\mathrm{S}} / G_{\mathrm{S}}} \sum_{q=0}^{\infty} s_{n}(t) c_{n}\left(t-q T_{s}^{\mathrm{S}}\right)$, the corresponding symbols received at the $k$ th relay $r^{k}(t)$ and at the destination $r^{\mathrm{SD}}(t)$ are written as: $r^{k}(t) \stackrel{=}{=}$ $\sum_{n=1}^{N} h_{n}^{\mathrm{SR}_{k}} y_{n}\left(t-\tau_{n}\right) e^{j\left(2 \pi f_{c} t+\phi_{n}^{\mathrm{SR}_{k}}\right)}+n_{k}(t)$ and $r^{\mathrm{SD}}(t)=$ $\sum_{n=1}^{N} h_{n}^{\mathrm{SD}} y_{n}\left(t-\tau_{n}\right) e^{j\left(2 \pi f_{c} t+\phi_{n}^{\mathrm{SD}}\right)}+n_{\mathrm{SD}}(t)$, where we have $\phi_{n}^{\mathrm{SR}=1}=\theta_{n}^{\mathrm{SR}}-2 \pi f_{c} \tau_{n}$ and $\phi_{n}^{\mathrm{SD}}=\theta_{n}^{\mathrm{SD}}-2 \pi f_{c} \tau_{n}$, each given by the uniformly distributed random phase, while $f_{c}$ is the operating carrier frequency. The noise components $n_{k}(t)$ of the $k$ th relay and of the destination, namely $n_{\mathrm{SD}}(t)$ has a zero mean and variances of $N_{0 k}$ and $N_{0 \mathrm{D}}$. Furthermore, $P_{\mathrm{S}}$ represents the transmission power of the source node, while $P_{\mathrm{R}} / 4=\left(1-P_{\mathrm{S}}\right) / 4$ is that of each relay node, where the total transmission power is considered to be unity.

\section{B. Relayed Signal}

During the cooperative phase of Fig. 1, the $N_{\mathrm{R}}=4$ relay nodes amplify-and-forward the received signals to the destination node with the aid of the STS scheme. More specifically, each relay node constructs the STS codeword based on the preassigned four spreading codes $c_{n}^{l}(t)(l=1,2,3,4)$, corresponding to the $n$th source, which are expressed as $c_{n}^{l}(t)=\sum_{i=0}^{L_{\mathrm{R}}-1} c_{n i}^{l} \psi_{T_{c}}\left(t-i T_{c}\right)$, where $L_{\mathrm{R}}$ is the code length of the spreading code and the symbol duration of the cooperating phase is given by $T_{s}^{\mathrm{R}}=L_{\mathrm{R}} T_{c}$. Let us furthermore define the spreading factor of the codes $c_{n}^{l}(t)$ as $G_{\mathrm{R}}$.

First, the $k$ th relay node despreads the received signal $r^{k}(t)$ with the aid of the classic correlation operation using the spreading code $c_{n}(t)$, in order to obtain the despread symbol $\bar{d}_{n}^{\mathrm{SR}_{k}}(q)$ corresponding to the transmitted symbols $\bar{v}_{n}(q)$, which is given by

$$
\begin{aligned}
\bar{d}_{n}^{\mathrm{SR}_{k}}(q)= & \frac{1}{T_{c} \sqrt{G_{\mathrm{S}}}} \int_{q T_{s}^{\mathrm{S}}+\tau_{n}}^{(q+1) T_{s}^{\mathrm{S}}+\tau_{n}} r^{k}(t) c_{n}\left(t-\tau_{n}\right) \\
& \times e^{-j\left(2 \pi f_{c} t+\phi_{n}^{\mathrm{SR}_{k}}\right)} d t \\
= & \sqrt{P_{\mathrm{S}}} h_{n}^{\mathrm{SR}_{k}} \bar{v}_{n}(q)+J_{n}^{\mathrm{SR}_{k}}(q)+N_{n}^{\mathrm{SR}_{k}}(q)
\end{aligned}
$$

where $J_{n}^{\mathrm{SR}_{k}}(q)$ is the Multi-User Interference (MUI)-related term induced by the other source nodes' signals

$$
\begin{aligned}
J_{n}^{\mathrm{SR}_{k}}(q)= & \frac{1}{T_{c}} \sum_{n^{\prime} \neq n} \sum_{q=0}^{\infty} \frac{\sqrt{P_{\mathrm{S}}}}{G_{\mathrm{S}}} h_{n^{\prime}}^{\mathrm{SR}_{k}} e^{j\left(\phi_{n^{\prime}}^{\mathrm{SR}_{k}}-\phi_{n}^{\mathrm{SR}_{k}}\right)} \\
& \times \int_{q T_{s}^{\mathrm{S}}+\tau_{n}}^{(q+1) T_{s}^{\mathrm{S}}+\tau_{n}} s_{n^{\prime}}\left(t-\tau_{n^{\prime}}\right) \\
& \times c_{n^{\prime}}\left(t-q T_{s}^{\mathrm{S}}-\tau_{n^{\prime}}\right) c_{n}\left(t-\tau_{n}\right) d t
\end{aligned}
$$

and $N_{n}^{\mathrm{SR}_{k}}(q)$ is a Gaussian distributed complex variable having a zero mean and a variance of $N_{0 k}$.

Let us then S/P convert the despread symbols $\bar{d}_{n}^{\mathrm{SR}_{k}}(q)$ to generate the block-indexed vector $\boldsymbol{d}_{n}^{\mathrm{SR}_{k}}(i)=\left[d_{n}^{\mathrm{SR}_{k} 1}(i), \cdots, d_{n}^{\mathrm{SR}_{k} 4}(i)\right]^{T}$ with the aid of the relation $d_{n}^{\mathrm{SR}_{k} l}(i)=\bar{d}_{n}^{\mathrm{SR}_{k}}(4 i+l-1)$. Furthermore, the time domain waveform of the despread symbols $d_{n}^{\mathrm{SR}_{k} l}(i)$ is given by $\hat{s}_{n}^{k l}(t)=\sum_{i=-\infty}^{\infty} d_{n}^{\mathrm{SR}_{k} l}(i) \psi_{T_{s}^{\mathrm{R}}}\left(t-i T_{s}^{\mathrm{R}}\right)$.

Finally, the $k$ th relay constructs the following STS-related signals $y_{n}^{k}(t)$ with the aid of the STS mapping block of Fig. 1, which are given by

$$
\begin{aligned}
y_{n}^{1}(t)= & \frac{\alpha_{1}}{\sqrt{G_{\mathrm{R}}}} \sum_{q=0}^{\infty}\left\{\hat{s}_{n}^{11}(t) c_{n}^{1}\left(t-q T_{s}^{\mathrm{R}}\right)\right. \\
& -\hat{s}_{n}^{12}(t) c_{n}^{2}\left(t-q T_{s}^{\mathrm{R}}\right) \\
& \left.-\hat{s}_{n}^{13}(t) c_{n}^{3}\left(t-q T_{s}^{\mathrm{R}}\right)-\hat{s}_{n}^{14}(t) c_{n}^{4}\left(t-q T_{s}^{\mathrm{R}}\right)\right\} \\
y_{n}^{2}(t)= & \frac{\alpha_{2}}{\sqrt{G_{\mathrm{R}}}} \sum_{q=0}^{\infty}\left\{\hat{s}_{n}^{22}(t) c_{n}^{1}\left(t-q T_{s}^{\mathrm{R}}\right)\right. \\
& +\hat{s}_{n}^{21}(t) c_{n}^{2}\left(t-q T_{s}^{\mathrm{R}}\right)+\hat{s}_{n}^{24}(t) c_{n}^{3}\left(t-q T_{s}^{\mathrm{R}}\right) \\
& \left.-\hat{s}_{n}^{23}(t) c_{n}^{4}\left(t-q T_{s}^{\mathrm{R}}\right)\right\}
\end{aligned}
$$




$$
\begin{aligned}
y_{n}^{3}(t)= & \frac{\alpha_{3}}{\sqrt{G_{\mathrm{R}}}} \sum_{q=0}^{\infty}\left\{\hat{s}_{n}^{33}(t) c_{n}^{1}\left(t-q T_{s}^{\mathrm{R}}\right)\right. \\
& -\hat{s}_{n}^{34}(t) c_{n}^{2}\left(t-q T_{s}^{\mathrm{R}}\right)+\hat{s}_{n}^{31}(t) c_{n}^{3}\left(t-q T_{s}^{\mathrm{R}}\right) \\
& \left.+\hat{s}_{n}^{32}(t) c_{n}^{4}\left(t-q T_{s}^{\mathrm{R}}\right)\right\} \\
y_{n}^{4}(t)= & \frac{\alpha_{4}}{\sqrt{G_{\mathrm{R}}}} \sum_{q=0}^{\infty}\left\{\hat{s}_{n}^{44}(t) c_{n}^{1}\left(t-q T_{s}^{\mathrm{R}}\right)\right. \\
& +\hat{s}_{n}^{43}(t) c_{n}^{2}\left(t-q T_{s}^{\mathrm{R}}\right)-\hat{s}_{n}^{42}(t) c_{n}^{3}\left(t-q T_{s}^{\mathrm{R}}\right) \\
& \left.+\hat{s}_{n}^{41}(t) c_{n}^{4}\left(t-q T_{s}^{\mathrm{R}}\right)\right\}
\end{aligned}
$$

with the aid of the normalization factor $\alpha_{k}=$ $\sqrt{P_{\mathrm{R}} /\left(4\left(P_{\mathrm{S}} \sigma_{\mathrm{SR}_{k}}^{2}+N_{0 k}\right)\right)}$. As a result of the quasi-simultaneous cooperative transmission of the STS codeword of (5)-(8), the corresponding received symbols at the destination node $r^{\mathrm{RD}}(t)$ can be expressed as $r^{\mathrm{RD}}(t)=$ $\sum_{n=1}^{N} \sum_{k=1}^{4} h_{n}^{\mathrm{RD}{ }_{k}} y_{n}^{k}\left(t-\tau_{n k}\right) e^{j\left(2 \pi f_{c} t+\phi_{n}^{\mathrm{RD}}\right)}+n_{\mathrm{RD}}(t)$, where $\phi_{n}^{\mathrm{RD}}{ }_{k}=\theta_{n}^{\mathrm{RD} \mathrm{D}_{k}}-2 \pi f_{c} \tau_{n k}$ and $n_{\mathrm{RD}}(t)$ has a zero mean and a variance of $N_{0 \mathrm{D}}$.

\section{CDSTS Detection Algorithm}

At the destination node of Fig. 1, the symbols transmitted from the $n$th source node are detected in a low-complexity manner so that a high diversity gain is achieved without any CSI estimation. First, the signals received at the broadcast phase $r^{\mathrm{SD}}(t)$ are despread as follows:

$$
\begin{aligned}
\bar{d}_{n}^{\mathrm{SD}}(q)= & \frac{1}{T_{c} \sqrt{G_{\mathrm{S}}}} \int_{q T_{s}^{\mathrm{S}}+\tau_{n}}^{(q+1) T_{s}^{\mathrm{S}}+\tau_{n}} r^{\mathrm{SD}}(t) c_{n}\left(t-\tau_{n}\right) \\
& \times e^{-j\left(2 \pi f_{c} t+\phi_{n}^{\mathrm{SD}}\right)} d t \\
= & \sqrt{P_{\mathrm{S}}} h_{n}^{\mathrm{SD}} \bar{v}_{n}(q)+J_{n}^{\mathrm{SD}}(q)+N_{n}^{\mathrm{SD}}(q)
\end{aligned}
$$

where we have the MUI-related component $J_{n}^{\mathrm{SD}}(q)$ :

$$
\begin{aligned}
J_{n}^{\mathrm{SD}}(q)= & \frac{1}{T_{c}} \sum_{n^{\prime} \neq n} \sum_{q=0}^{\infty} \frac{\sqrt{P_{\mathrm{S}}}}{G_{\mathrm{S}}} h_{n^{\prime}}^{\mathrm{SD}} e^{j\left(\phi_{n^{\prime}}^{\mathrm{SD}}-\phi_{n}^{\mathrm{SD}}\right)} \\
& \times \int_{q T_{s}^{\mathrm{S}}+\tau_{n}}^{(q+1) T_{s}^{\mathrm{S}}+\tau_{n}} s_{n^{\prime}}\left(t-\tau_{n^{\prime}}\right) \\
& \times c_{n^{\prime}}\left(t-q T_{s}^{\mathrm{S}}-\tau_{n^{\prime}}\right) c_{n}\left(t-\tau_{n}\right) d t
\end{aligned}
$$

while the AWGN-related component $N_{n}^{\mathrm{SD}}(q)$ is a Gaussian distributed complex variable having a zero mean and a variance of $N_{\mathrm{OD}}$. For simplicity of the treatment, the despread symbols $\bar{d}_{n}^{\mathrm{SD}}(q)$ are then rearranged at the detector into the following vectorial form

$$
\begin{aligned}
\xi_{n}^{\mathrm{SD}}(i) & =\left[\xi_{n}^{\mathrm{SD}_{1}}(i) \xi_{n}^{\mathrm{SD}_{2}}(i) \xi_{n}^{\mathrm{SD}_{3}}(i) \xi_{n}^{\mathrm{SD}_{4}}(i)\right]^{T} \\
& =\frac{1}{\sqrt{4}}\left[\begin{array}{c}
d_{n}^{\mathrm{SD}_{1}}(i)+d_{n}^{\mathrm{SD}_{2}}(i)+d_{n}^{\mathrm{SD}_{3}}(i)+d_{n}^{\mathrm{SD}_{4}}(i) \\
-d_{n}^{\mathrm{SD}_{2}}(i)+d_{n}^{\mathrm{SD}_{1}}(i)-d_{n}^{\mathrm{SD}_{4}}(i)+d_{n}^{\mathrm{SD}_{3}}(i) \\
-d_{n}^{\mathrm{SD}_{3}}(i)+d_{n}^{\mathrm{SD}_{4}}(i)+d_{n}^{\mathrm{SD}_{1}}(i)-d_{n}^{\mathrm{SD}_{2}}(i) \\
-d_{n}^{\mathrm{SD}_{4}}(i)-d_{n}^{\mathrm{SD}_{3}}(i)+d_{n}^{\mathrm{SD}_{2}}(i)+d_{n}^{\mathrm{SD}_{1}}(i)
\end{array}\right]
\end{aligned}
$$

with the aid of the symbol-to-block transformation $d_{n}^{\mathrm{SD}_{l}}(i)=$ $\bar{d}_{n}^{\mathrm{SD}}(4 i+l-1)$. Next, the signals received during the cooperative phase $r^{\mathrm{RD}}(t)$ are despread to form $\xi_{n}^{\mathrm{RD}}(i)=$ $\left[\xi_{n}^{\mathrm{RD}_{1}}(i) \xi_{n}^{\mathrm{RD}_{2}}(i) \xi_{n}^{\mathrm{RD}_{3}}(i) \xi_{n}^{\mathrm{RD}_{4}}(i)\right]^{T}$, which is given by

$$
\begin{aligned}
\xi_{n}^{\mathrm{RD}}(i)= & \frac{1}{T_{c} \sqrt{G_{\mathrm{R}}}} \sum_{k=1}^{4} \int_{i T_{s}^{\mathrm{R}}+\tau_{n k}}^{(i+1) T_{s}^{\mathrm{R}}+\tau_{n k}} r^{\mathrm{RD}}(t) \\
& \times c_{n}^{l}\left(t-\tau_{n k}\right) e^{-j\left(2 \pi f_{c} t+\phi_{n}^{\mathrm{RD}_{k}}\right)} d t \\
= & D_{n}^{\mathrm{RD}}(i)+J_{n}^{\mathrm{RD}}(i)+N_{n}^{\mathrm{RD}_{l}}(i)
\end{aligned}
$$

where $D_{n}^{\mathrm{RD}}(i)=\left[D_{n}^{\mathrm{RD}_{1}}(i) D_{n}^{\mathrm{RD}_{2}}(i) D_{n}^{\mathrm{RD}_{3}}(i) D_{n}^{\mathrm{RD}_{4}}(i)\right]^{T}$ can be expressed as $D_{n}^{\mathrm{RD}}(i)=\Xi(i) \chi_{n}$ with the relations of

$$
\begin{aligned}
\Xi(i)= & {\left[\begin{array}{cccc}
d_{n}^{\mathrm{SR}_{1} 1}(i) & d_{n}^{\mathrm{SR}_{2} 2}(i) & d_{n}^{\mathrm{SR}_{3} 3}(i) & d_{n}^{\mathrm{SR}_{4} 4}(i) \\
-d_{n}^{\mathrm{SR}_{1} 2}(i) & d_{n}^{\mathrm{SR}_{2} 1}(i) & -d_{n}^{\mathrm{SR}_{3} 4}(i) & d_{n}^{\mathrm{SR}_{4} 3}(i) \\
-d_{n}^{\mathrm{SR}_{1} 3}(i) & d_{n}^{\mathrm{SR}_{2} 4}(i) & d_{n}^{\mathrm{SR}_{3} 1}(i) & -d_{n}^{\mathrm{SR}_{4} 2}(i) \\
-d_{n}^{\mathrm{SR}_{1} 4}(i) & -d_{n}^{\mathrm{SR}_{2} 3}(i) & d_{n}^{\mathrm{SR}_{3} 2}(i) & d_{n}^{\mathrm{SR}_{4} 1}(i)
\end{array}\right] } \\
& \chi_{n}=\left[\alpha_{1} h_{n}^{\mathrm{RD}_{1}} \alpha_{2} h_{n}^{\mathrm{RD}_{2}} \alpha_{3} h_{n}^{\mathrm{RD}_{3}} \alpha_{4} h_{n}^{\mathrm{RD}_{4}}\right]^{T}
\end{aligned}
$$

and $J_{n}^{\mathrm{RD}}(i)$ is the corresponding MUI component. Furthermore, the noise component $N_{n}^{\mathrm{RD}}(i)$ is a Gaussian distributed complex variable having a zero mean and a variance of $4 N_{0 \mathrm{D}}$. According to [10], let us denote the rearranged vectorial form of the despread signals as follows:

$$
\begin{aligned}
\zeta_{n}^{X_{1}}(i) & =\left[\begin{array}{llll}
\xi_{n}^{X_{1}}(i) & \xi_{n}^{X_{2}}(i) & \xi_{n}^{X_{3}}(i) & \xi_{n}^{X_{4}}(i)
\end{array}\right]^{T} \\
\zeta_{n}^{X_{2}}(i) & =\left[\begin{array}{llll}
-\xi_{n}^{X_{2}}(i) & \xi_{n}^{X_{1}}(i) & \xi_{n}^{X_{4}}(i) & -\xi_{n}^{X_{3}}(i)
\end{array}\right]^{T} \\
\zeta_{n}^{X_{3}}(i) & =\left[\begin{array}{llll}
-\xi_{n}^{X_{3}}(i) & -\xi_{n}^{X_{4}}(i) & \xi_{n}^{X_{1}}(i) & \xi_{n}^{X_{2}}(i)
\end{array}\right]^{T} \\
\zeta_{n}^{X_{4}}(i) & =\left[\begin{array}{llll}
-\xi_{n}^{X_{4}}(i) & \xi_{n}^{X_{3}}(i) & -\xi_{n}^{X_{2}}(i) & \xi_{n}^{X_{1}}(i)
\end{array}\right]^{T}
\end{aligned}
$$

where we have $X=\mathrm{SD}$ or RD. Finally, based on (15)-(18), the detector evaluates

$$
\begin{aligned}
\Re & \left.\zeta_{n}^{\mathrm{SD}_{1}{ }^{T}}(i+1) \zeta_{n}^{\mathrm{SD}_{l}}{ }^{*}(i)+\zeta_{n}^{\mathrm{RD}_{1}{ }^{T}}(i+1) \zeta_{n}^{\mathrm{RD}_{l}}{ }^{*}(i)\right] \\
= & 4 P_{\mathrm{S}}\left\{\left(h_{n}^{\mathrm{SD}}\right)^{2}+\sum_{k=1}^{4}\left(\alpha_{k} h_{n}^{\mathrm{SR}_{k}} h_{n}^{\mathrm{RD}_{k}}\right)^{2}\right\} \\
& \times\left\|\boldsymbol{v}_{n}(i)\right\| a_{n}^{l}(i)+J_{n}(i)+N_{n}(i)
\end{aligned}
$$

where $J_{n}(i)$ represents the MUI-related term, originated from $J_{n}^{\mathrm{SR}}, J_{n}^{\mathrm{SD}}$, and $J_{n}^{\mathrm{RD}}$, while $N_{n}(i)$ is the AWGN-related term obeying $N_{n}^{\mathrm{SR}_{k}}$ in (3), $N_{n}^{\mathrm{SD}}$ in (10) and $N_{n}^{\mathrm{RD}}$ in (13). Finally, the symbols $a_{n}^{l}(i)$ are detected by the low-complexity hard decision decoder evaluating (19).

\section{BASIC PROPERTIES OF LS CODES}

As represented by the term $J_{n}(i)$ in (19), the attainable performance of our CDSTS system is affected by the MUI, therefore dependent on the spreading codes employed. In order to mitigate the performance degradation due to the MUI, we employ LS codes as the spreading codes in our CDSTS system, which exhibit a so-called Interference Free Window (IFW), where the off-peak aperiodic auto-correlation values as well as the aperiodic cross-correlation values become zero, resulting in zero ISI and zero MAI, provided that the maximum delay of the asynchronous transmissions is within the width of the IFW.

Here we only highlight the basic characteristics and the parameters of the LS codes employed, since the design of LS codes was detailed for example in [9] and in the references therein. To be specific, LS codes are constructed with the aid of a $\left(P_{\mathrm{LS}} \times P_{\mathrm{LS}}\right)$-dimensional Walsh-Hadamard matrix as well as an orthogonal complementary code set of length $N_{\mathrm{LS}}$. More specifically, by inserting $W_{0}$ zeros both at the beginning and in the center of the complementary code pair, we can generate 
$P_{\mathrm{LS}}$ LS codes having an IFW of $\min \left\{N_{\mathrm{LS}}-1, W_{0}\right\}$ chip durations, where the corresponding code length of the LS codes is $L=N_{\mathrm{LS}} P_{\mathrm{LS}}+2 W_{0}$. According to [9], the parameter-based notation of $\mathrm{LS}$ codes is given by $\mathrm{LS}\left(N_{\mathrm{LS}}, P_{\mathrm{LS}}, W_{0}\right) .{ }^{1}$

\section{PERFORMANCE AND CONCLUSION}

We consider a BPSK-modulated CDSTS system supporting $N=4$ source nodes, each of which is supported by $N_{\mathrm{R}}=4$ source-specific relay nodes that are allocated prior to communications. The synchronization delays $\tau_{n}$ of the source nodes and those of the associated $k$ th relay nodes $\tau_{n k}$ are uniformly distributed in $\left[0, \tau_{\max }\right]$, where $\tau_{\max }$ is the maximum delay value, ranging from 0 to $8 T_{c}$ in our simulations. We assumed that all the channels were nondispersive Rayleigh fading channels, while considering the geometrical gains of $\sigma_{\mathrm{SR}_{k}}^{2}=8, \sigma_{\mathrm{RD}_{k}}^{2}=$ 2 and $\sigma_{\mathrm{SD}}^{2}=1$ for the simplified treatment of the relay selection. As mentioned above, LS codes are employed as our DS-CDMA spreading codes, where the source nodes have the LS codes of $\mathrm{LS}\left(N_{\mathrm{LS}}, P_{\mathrm{LS}}, W_{0}\right)=\mathrm{LS}(8,4,7)$, while the relay nodes employ $\mathrm{LS}\left(N_{\mathrm{LS}}, P_{\mathrm{LS}}, W_{0}\right)=\mathrm{LS}(8,16,7)$, indicating that the corresponding width of the IFW is $7 T_{c}$. Furthermore, the power allocated to the souce and to the relay nodes was set to $P_{\mathrm{S}}=$ $P_{\mathrm{R}}=0.5$, as suggested in [4]. Fig. 2(a) shows the achievable BER performance of our CDSTS system in conjunction with the different maximum delay values of $\tau_{\max }=T_{c}, 2 T_{c}, 4 T_{c}, 7 T_{c}$ and $8 T_{c}$. In addition to the above-mentioned LS codes, Gold codes are also considered as spreading codes in order to show the effects of diverse spreading codes on the achievable performance. Furthermore, we also characterized the noncooperative differential transmission scenario associated with $P_{\mathrm{S}}=1$, where the source nodes communicate with the destination node without relying on any cooperating nodes. Observe in Fig. 2(a) that for the case of $\tau_{\max } \leq 7 T_{c}=W_{0}$, the LS code-based CDSTS system attained a high diversity gain, outperforming the noncooperative scenario, although an error floor was exhibited for the high-delay scenario of $\tau_{\max }=8 T_{c}>W_{0}$ due to the effects of the unsuppressed interfering signal components arriving outside the IFW. This also implies that the relays' synchronization error-induced degradations were overcome, while dispensing with the typical simplfiying assumption of conventional CDSTC schemes, when assuming perfectly-synchronized relay nodes [5].

Although the aforementioned systems assumed the presence of frequency-flat channels, our CDSTS system can be readily employed for transmission over frequency-selective channels by introducing the concept of RAKE combining in (19). Fig. 2(b) shows the achievable BER performance of our CDSTS in a frequency-selective channel environment in conjunction with $\nu=1,2$ and 4 RAKE fingers, where the maximum delay value was set to $\tau_{\max }=3 T_{c}$. Furthermore, we considered a correlated tapped delay line channel model for each channel, where the number of CIR taps was $L_{\text {tap }}=4$ and the correlation factor $\rho_{i j}$ between the $i$ th tap and the $j$ th tap was set to $\rho_{i j}=$ 0.5 . For comparison, we also simulated the noncooperative differentially-encoded transmission scheme using LS codes and

\footnotetext{
${ }^{1}$ Note that while the size of the differentially-encoded symbol blocks $\boldsymbol{v}_{n}$ is chosen to be identical to the number of relay nodes $N_{\mathrm{R}}$, the parameter $P_{\mathrm{LS}}$ has to satisfy the condition of $P_{\mathrm{LS}} \geq N$ for the source nodes as well as $P_{\mathrm{LS}} \geq N \times$ $N_{\mathrm{R}}$ for the relay nodes, indicating that increasing the number of source nodes per time slot $N$ has the following consequences: the number of cooperative nodes $N_{\mathrm{R}}$ and the LS codes' IFW width $W_{0}$, therefore also the number of chips in the LS codes increases. Consequently also the effective bit rate of the CDMA system is decreased in a fixed-bandwidth scenario.
}

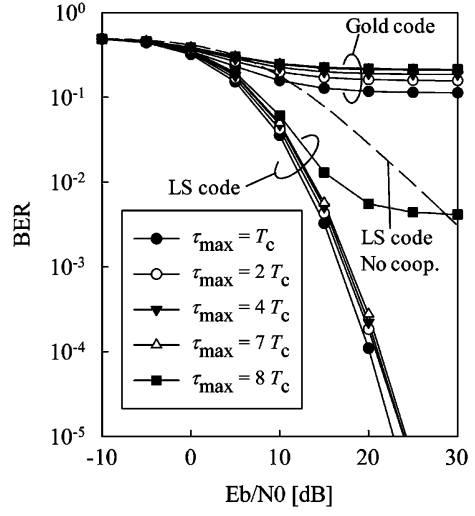

(a)

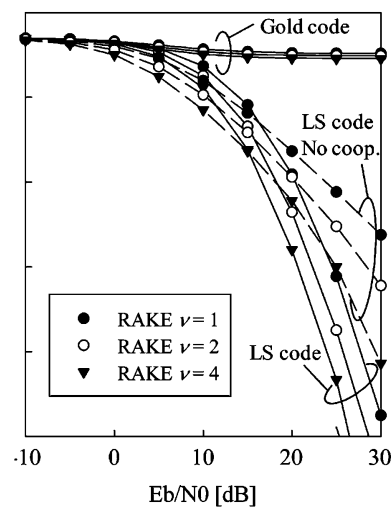

(b)
Fig. 2. Achievable BER performance of our CDSTS system.

the Gold code-based CDSTS benchmark scheme. It is seen in Fig. 2(b) that both our LS code-based CDSTS arrangement and the noncooperative scenario using LS codes benefitted from an increased path diversity gain upon increasing the number of RAKE fingers $\nu$ owing to the ideal auto-correlation characteristics of the LS codes. More specifically, our LS code-based CDSTS is capable of gleaning both space-time diversity gain as well as path diversity gain.

In this contribution we proposed a practical CDSTC protocol, where neither channel estimation nor symbol-level synchronization is required at the nodes. Our simulation results demonstrate that the proposed CDSTS scheme is capable of combating the effects of asynchronous transmissions, provided that the maximum synchronization delay of the relay nodes is within the width of the IFW.

\section{REFERENCES}

[1] J. Laneman and G. Wornell, "Distributed space-time-coded protocols for exploiting cooperative diversity in wireless networks," IEEE Trans. Inform. Theory, vol. 49, no. 10, pp. 2415-2425, 2003.

[2] B. Hughes, "Differential space-time modulation," IEEE Trans. Inform. Theory, vol. 46, no. 7, pp. 2567-2578, 2000.

[3] P. Tarasak, H. Minn, and V. Bhargava, "Differential modulation for two-user cooperative diversity systems," IEEE J. Sel. Areas Commun., vol. 23, no. 9, pp. 1891-1900, 2005.

[4] Y. Jing and H. Jafarkhani, "Distributed differential space-time coding for wireless relay networks," IEEE Trans. Commun., vol. 56, no. 7, pp. 1092-1100, 2008.

[5] R. C. Palat, A. Annamalai, and J. H. Reed, "Accurate bit-error-rate analysis of bandlimited cooperative OSTBC networks under timing synchronization errors," IEEE Trans. Veh. Technol., vol. 58, no. 5, pp. 2191-2200, 2009.

[6] X. Li, "Space-time coded multi-transmission among distributed transmitters without perfect synchronization," IEEE Signal Process. Lett., vol. 11, no. 12, pp. 948-951, 2004.

[7] X. Guo and X. Xia, "A distributed space-time coding in asynchronous wireless relay networks," IEEE Trans. Wireless Commun., vol. 7, no. 5, pp. 1812-1816, 2008.

[8] L. Hanzo, L.-L. Yang, E.-L. Kuan, and K. Yen, Single and Multi-Carrier CDMA: Multi-User Detection, Space-Time Spreading, Synchronisation and Standards. Hoboken, NJ: Wiley, 2003.

[9] H. Wei, L. Yang, and L. Hanzo, "Interference-free broadband single-and multicarrier DS-CDMA," IEEE Commun. Mag., vol. 43, no. 2, pp. 68-73, 2005.

[10] M. El-Hajjar, O. Alamri, S. X. Ng, and L. Hanzo, "Turbo detection of precoded sphere packing modulation using four transmit antennas for differential space-time spreading," IEEE Trans. Wireless Commun., vol. 7, no. 3, pp. 943-952, 2008. 